EEG of relatives showed elevated incidences of spike and wave and photosensitivity in both groups. However, in parents of the non-GTCS group, EEG abnormality was more frequent than in parents of the GTCS group. Mothers' EEG was the best predictor of seizure risk in probands' siblings. Early childhood epilepsy with absences overlaps with early onset GTCS and myoclonic astatic epilepsy on one hand and with childhood absence epilepsy on the other. This syndrome could not be regarded as a specific entity. The analysis supports the assumption of heterogeneity within early childhood absence epilepsy. (Doose H. Absence epilepsy of early chidhood - genetic aspects. Eur I Pediatr May 1994;153:372-377). (Respond: Dr H Doose, Norddeutsches Epilepsie-Zentrum, D-24223 Raisdorf, Germany).

COMMENT. Three type of absence epilepsy are distinguished by the International Classification: 1) childhood absence with absence as presenting symptom, 2) juvenile absence with or without GTCS, and 3) epilepsy with myoclonic absences. Absence epilepsy of early childhood has not been distinguished as a specific entity, although Doose has demonstrated some characteristics which might allow differentiation from childhood absence. These include: onset before 5 years of age with absences or GTCS, male preponderance, associated myoclonic and/or myoclonic astatic seizures, poor response to AEDs, and unfavorable psychologic and social development in children with GTCS. The clinical and family data reported here are considered to support genetic heterogeneity within the disorder. Both non-GTCS and GTCS forms of the syndrome appear to be part of the larger spectrum of idiopathic generalized epilepsies of childhood.

\title{
GABA SYNTHESIS AND PYRIDOXINE SEIZURES
}

A reduction in pyridoxal-5-phosphate (PLP) dependent enzyme, glutamic acid decarboxylase (GAD), which synthesizes GABA, is reported in a 3 month-old infant with seizures responsive to pyridoxine treated at University of California, Davis. The infant had asynchronous jerking of arms and legs and lip smacking at birth which responded to phenobarbital and phenytoin. MRI showed enlarged ventricles, and the EEG demonstrated bitemporal and left frontal epileptiform activity. Seizures recurred at 7 weeks and were not responsive to phenobarbital and carbamazepine. When admitted at 3 months, $100 \mathrm{mg}$ pyridoxine IV stopped a seizure within 5 minutes. Anticonvulsants were discontinued and electrographic and clinical seizure activity was controlled with oral pyridoxine $25 \mathrm{mg}$ daily. PLP independent GAD activities measured in skin fibroblasts of the patient and 5 controls were similar, whereas the patient's PLP dependent GAD activity was reduced. (Gospe SM Jr, et al. Reduced GABA synthesis in pyridoxine-dependent seizures. Lancet May 7 1994;343:1133-34). (Respond: Dr Sidney M Gospe Jr, Division of Child Neurology, University of California, Davis Medical Center, 2315 Stockton Boulevard, Sacramento, CA 95817, USA).

COMMENT. An alteration in the function of PLP dependent GAD appears to be responsible for the autosomal recessive syndrome of pyridoxine dependent epilepsy. 